\title{
Direct Observation of the Statics and Dynamics of Emergent Magnetic Monopoles in a Chiral Magnet
}

\author{
N. Kanazawa๑ ${ }^{1,{ }^{*}}$ A. Kitaori, ${ }^{1,{ }^{*}}$ J. S. White $\odot,{ }^{2, *}$ V. Ukleev $\odot,{ }^{2}$ H. M. Rønnow, ${ }^{3}$ A. Tsukazaki, ${ }^{4}$ \\ M. Ichikawa, ${ }^{1}$ M. Kawasaki, ${ }^{1,5}$ and Y. Tokura ${ }^{1,5,6}$ \\ ${ }^{1}$ Department of Applied Physics, University of Tokyo, Tokyo 113-8656, Japan \\ ${ }^{2}$ Laboratory for Neutron Scattering and Imaging (LNS), Paul Scherrer Institute (PSI), CH-5232 Villigen, Switzerland \\ ${ }^{3}$ Laboratory for Quantum Magnetism (LQM), Institute of Physics, École Polytechnique Fédérale de Lausanne (EPFL), \\ CH-1015 Lausanne, Switzerland \\ ${ }^{4}$ Institute for Materials Research, Tohoku University, Sendai 980-8577, Japan \\ ${ }^{5}$ RIKEN Center for Emergent Matter Science (CEMS), Wako 351-0198, Japan \\ ${ }^{6}$ Tokyo College, University of Tokyo, Tokyo 113-8656, Japan
}

(Received 21 April 2020; accepted 24 August 2020; published 25 September 2020)

\begin{abstract}
In the three-dimensional (3D) Heisenberg model, topological point defects known as spin hedgehogs behave as emergent magnetic monopoles, i.e., quantized sources and sinks of gauge fields that couple strongly to conduction electrons, and cause unconventional transport responses such as the gigantic Hall effect. We observe a dramatic change in the Hall effect upon the transformation of a spin hedgehog crystal in a chiral magnet MnGe through combined measurements of magnetotransport and small-angle neutron scattering (SANS). At low temperatures, well-defined SANS peaks and a negative Hall signal are each consistent with expectations for a static hedgehog lattice. In contrast, a positive Hall signal takes over when the hedgehog lattice fluctuates at higher temperatures, with a diffuse SANS signal observed upon decomposition of the hedgehog lattice. Our approach provides a simple way to both distinguish and disentangle the roles of static and dynamic emergent monopoles on the augmented Hall motion of conduction electrons.
\end{abstract}

DOI: 10.1103/PhysRevLett.125.137202

Some topological orders in condensed matter systems behave analogously to elementary particles, enabling a study of their electrodynamics in the realm of low-energy physics. As a representative example, artificial magneticmonopole-like structures have been proposed in different ways [1-8]. A hedgehog-type spin structure coupled with conduction electrons in a metal $[2,6]$ can take on a quantized nature like a magnetic monopole. Such structures are composed of spins pointing in to or out from a singular point, and carry integer topological charges $Q=(1 / 8 \pi)$ $\epsilon^{i j k} \int_{S} d S_{k} \boldsymbol{n}(\boldsymbol{r}) \cdot\left[\partial_{i} \boldsymbol{n}(\boldsymbol{r}) \times \partial_{j} \boldsymbol{n}(\boldsymbol{r})\right]= \pm 1 \quad$ [9] [Fig. 1(a)]. This quantity represents the mapping of the spin moment $\boldsymbol{m}(\boldsymbol{r})$ on the sphere $S$ surrounding the singular point in real space to the sphere of the unit vector $\boldsymbol{n}(\boldsymbol{r})=$ $\boldsymbol{m}(\boldsymbol{r}) /|\boldsymbol{m}(\boldsymbol{r})|$. Meanwhile, conduction electrons couple to noncoplanar spins through the Berry curvature $b_{k}=$ $\frac{1}{2} \epsilon^{i j k} \boldsymbol{n}(\boldsymbol{r}) \cdot\left[\partial_{i} \boldsymbol{n}(\boldsymbol{r}) \times \partial_{j} \boldsymbol{n}(\boldsymbol{r})\right]$, which corresponds to an effective gauge field, a so-called emergent magnetic field (EMF), that affects electron motion [10]. Correspondingly, conduction electrons indeed regard hedgehogs as quantized sources or sinks of EMF with magnetic charge, $Q_{m}=$ $(1 / 4 \pi) \int_{S} d S_{k} b_{k}= \pm 1$ [Fig. 1(b)].

Spin hedgehogs are predicted to be thermally excited in 3D ferromagnetic states and be responsible for both the ferromagnetic-to-paramagnetic transition [11,12], and the concomitant enhancement of anomalous Hall effect (AHE) [13-17]. In analogy with the BerezinskiiKosterlitz-Thouless transition in the 2D $X Y$ model $[18,19]$ driven by the vortex pair dynamics, pairwise excitations of oppositely charged hedgehogs play a key role in mediating the disordering transition in the 3D Heisenberg spin system. With increasing density of the pairwise excitations in the vicinity of the transition, conduction electrons are strongly deflected by the quantum flux lines of EMF generated at skyrmion strings, which bridge the hedgehog pairs [Figs. 1(c) and 1(d)]. The Hall effect due to EMF attracts renewed attention and is termed the topological Hall effect (THE) [20]; typically it can be generated by a static order of skyrmion strings in chiral magnets [21-23].

While the THE serves as a probe of the static properties of emergent monopoles [13-17], their dynamics, which leads to fluctuations of EMF and hence scatters electrons, would manifest itself as small noise voltage $[6,24,25]$ posing considerable difficulty for its detection. It is nonetheless expected that spin fluctuations can cause skew scattering of electrons and show up as a steady Hall signal if they have finite thermally averaged total chirality $\left\langle\boldsymbol{S}_{1}\right.$. $\left.\left(\boldsymbol{S}_{2} \times \boldsymbol{S}_{3}\right)\right\rangle$ [26]. In this context, thermally excited hedgehogs displaying large spin chirality may produce distinct 


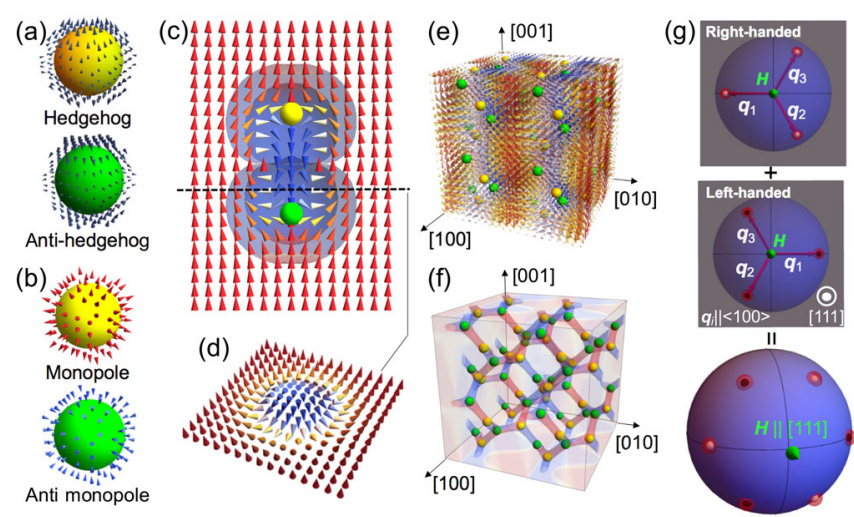

FIG. 1. (a),(b) Schematic illustrations of hedgehog and antihedgehog spin structures (a) and their monopolelike EMF distributions (b). (c) A pair of hedgehog (yellow dot) and antihedgehog (green dot) in the ferromagnetic background (red arrows). The magnetic line defect (blue arrows) appears between the pair, where the negative EMF emerges as indicated by the blue region. (d) The bridging skyrmionic structure between the pair in cross section. (e) Cubic hedgehog lattice (HL) state in MnGe. Reddish and bluish arrows indicate spins with positive and negative $z$ components, respectively. (f) EMF distribution of the HL at zero magnetic field. Reddish and bluish regions represent EMF with positive and negative $z$ components, respectively. (g) Expected neutron scattering intensity map for the MnGe thick film with the two chiral crystalline domains. Red dots represent magnetic reflections. White and black dots represent directions of $\langle 100\rangle$ crystalline axes of right- and lefthanded domains, respectively.

Hall signatures through preferential selection of one of their chiral fluctuation modes.

As an ideal platform for studying the emergentmonopole dynamics, we target the chiral magnet $\mathrm{MnGe}$ in which hedgehog pairs condense due to higherorder magnetic interactions [27-31], and form a hedge hog-antihedgehog lattice bonded by skyrmion strings [32-34] [Figs. 1(e) and 1(f)]. Owing to the lack of space inversion symmetry in $\mathrm{MnGe}$, we expect a homochiral nature of spin fluctuations [34]. From small-angle neutron scattering (SANS) on a thick epitaxial film of $\mathrm{MnGe}$ (see the Supplemental Material [35]), we identify thermal fluctuations of hedgehogs to develop at temperatures $(T \mathrm{~s})$ far below the magnetic transition temperature $\left(T_{N} \sim 170 \mathrm{~K}\right)$. In particular, we observe a pronounced fluctuating hedgehog state when the magnetic field $(H)$-driven transformation of the hedgehog lattice (HL) from cubic to rhombohedral takes place, and the rearrangement of hedgehogs are involved. Meanwhile, through electrical transport measurements on a bulk single crystal synthesized by a flux method under high pressure [35], we observe a distinct Hall response in the $T-H$ region of pronounced hedgehog fluctuation which is opposite in sign to the THE induced by EMF of the static HL. The close correlation between SANS data and Hall response profiles reveal charge transport characteristics that are distinct according to if they are due to the statics or dynamics of emergent monopoles.

We performed SANS on a MnGe film of 3- $\mu$ m thickness, which is roughly 1000 times larger than the magnetic modulation period of 3-6 $\mathrm{nm}[33,37]$. Due to reduced surface and interface effects, the magnetic structure in the film is confirmed to be essentially equivalent to that in the bulk. The HL state is constructed by the superposition of three orthogonal proper-screw structures with propagation vectors $\left(\boldsymbol{q}_{1}, \boldsymbol{q}_{2}\right.$, and $\left.\boldsymbol{q}_{3}\right)$ aligned with the equivalent $\langle 100\rangle$ directions $[32,34]$. Although the film is epitaxially grown along the [111] direction, there exist two crystalline domains with opposite chirality, which are related by a mirror operation with respect to the (111) plane [47]. As a result, not a threefold-, but instead a sixfold-symmetric pattern of scattering peaks appears around the [111] direction, along which $H$ is also applied [Fig. 1(g)]. Here we also note that there is controversy regarding the magnetic structure [35].

With varying $T$ and $H$, we investigated the peak positions and area sizes of SANS diffraction spots. Figures 2(a)-2(f) show representative scattering patterns and their schematic illustrations viewed from the film normal of [111] (see also Figs. S2-S4 [35]). In finite $H$, the $H$ direction remains fixed along [111], and both the sample and cryomagnet are rotated together to map the SANS intensity. Due to the limited opening angle of the magnet's neutron access windows, some azimuthal angle ranges [gray shaded regions in Figs. 2(d)-2(f)] are not observed. At zero magnetic field, the sample is rotated independently from the magnet, and the expected sixfoldsymmetric pattern of satellites due to cubic HL formation is indeed observed [Figs. 2(a)-2(c)]. The spot sizes are larger at $T=100 \mathrm{~K}$ compared to those at $T=2$ and $50 \mathrm{~K}$ as evident from Figs. 2(a)-2(c) and from quantitative evaluation of the spot area shown in Figs. 2(j)-2(1). This indicates a strong $T$ dependence of the magnetic correlation length deep within the ordered phase. Taking into account the results reported from so-called MIEZE spectroscopy on polycrystalline bulk samples [42], the observed spatial disorder of the magnetic structure can be understood as a consequence of the temporal spin fluctuations observed to survive over an exceptionally wide temperature range above $T=30 \mathrm{~K}$. In contrast, the spread in width of the magnetic Bragg peaks below $T=30 \mathrm{~K}$ is attributed mainly to static disorder of the hedgehog arrangement. With increasing $H$, the three $\boldsymbol{q}$ vectors of HL tilt from their original alignment with $\langle 100\rangle$ towards the $H$ direction [Figs. 2(d)-2(f)]. Eventually the magnetic diffraction spots from each of the crystalline domains tend towards overlapping, resulting in a threefold scattering pattern as deduced at, for example, $T=50 \mathrm{~K}$ and $\mu_{0} H=6 \mathrm{~T}$ [Fig. 2(e)]. Although a large portion of the scattering pattern is either inaccessible at $T=2 \mathrm{~K}$ and $\mu_{0} H=8 \mathrm{~T}$ [Fig. 2(d)], or the increasingly diffuse signal spreads 

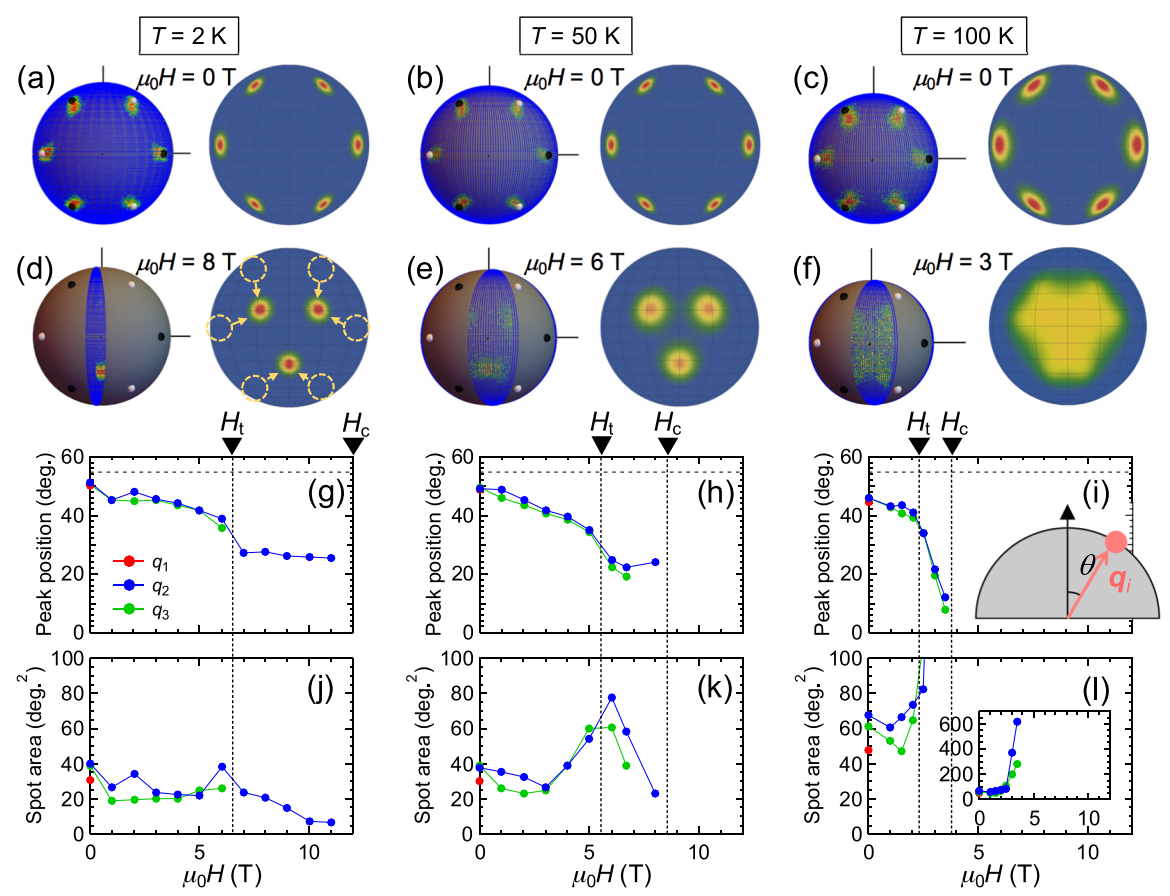

FIG. 2. (a)-(f) Representative SANS patterns (left panels) and their schematic illustrations (right panels) at zero (a)-(c) and finite (d)(f) magnetic fields. The view is along the film normal direction. (g)-(1), Magnetic-field dependence of positions and area sizes of magnetic Bragg satellites at various temperatures. The position and area size are described as the angle $\theta$ between $\boldsymbol{q}_{i}$ and the film normal [inset of (i)] and the product of half widths of the elliptical intensity distribution along its long and short axes, respectively. The inset of (l) shows an enlargement of the main panel. The horizontal dotted lines at $\theta=54^{\circ}$ represent the angle which corresponds to a $q$-vector alignment of spots with the original orthogonal $\langle 100\rangle$ axes, while vertical dotted lines indicate critical fields for HL transformation $\left(H_{t}\right)$ and ferromagnetic transition $\left(H_{c}\right)$.

beyond the observable range at $T=100 \mathrm{~K}$ [Fig. 2(f)], the overall scattering patterns at these $T$ and $H$ conditions also tend towards being threefold symmetric too. Since the directional change in the basis $\boldsymbol{q}$ vectors of the HL requires a lattice transformation from cubic to rhombohedral forms, hedgehog motion must be induced at the transition. Such a large deformation of the HL likely enhances hedgehog fluctuations, resulting in the diffuse scattering patterns observed under $H$ at 50 and $100 \mathrm{~K}$, which are temperatures far below $T_{N}$.

Next we measured the Hall resistivity $\rho_{y x}$ to reveal its connection to the magnetic state. To estimate the THE, which shows up in addition to the normal and anomalous Hall effects (NHE and AHE), we employed a complementary approach using both thick film and bulk single crystal samples. Since the inevitable small cracks in the thick film inhibit electrical transport measurements, we used the single crystal [35]. The single crystal was cut into a cuboid with a thickness $(t=3 \mu \mathrm{m})$ and the largest face being the (111) plane (Fig. S1 [35]). On the other hand, we exploited the thick film with larger volume than the single-crystal cuboid for magnetization $(M)$ measurements. Figures 3(d)-3(f) show the $H$ dependence of $\rho_{y x}$ and the estimated conventional Hall response $\rho_{y x}^{\text {est. }}=R_{0} H+R_{A} \rho_{x x}^{2} M$, where $R_{0}$ and $R_{A}$ are coefficients for the NHE and AHE, respectively. As is obvious from the difference between the $M-H$ [Figs. 3(a)-3(c)] and $\rho_{y x}-H$ [Figs. 3(d)-3(f)] curves, there are additional Hall contributions with various characteristics that are collectively expressed as $\rho_{y x}^{\mathrm{T}}$. At $T=10 \mathrm{~K}$, where the HL state is static, $\rho_{y x}$ shows a broad negative signal with a small positive bump structure below the ferromagnetictransition field $H_{c}$, before rising sharply and exceeding $\rho_{y x}^{\text {est }}$ with further increasing $H$ [Fig. 3(d)]. At $T=50 \mathrm{~K}$, where the HL state fluctuates upon its transformation by $H, \rho_{y x}$ overtakes $\rho_{y x}^{\text {est }}$ above $H=3 \mathrm{~T}$, peaking at $H=6 \mathrm{~T}$, and finally converges to $\rho_{y x}^{\text {est }}$. [Fig. 3(e)]. At $T=100 \mathrm{~K}$, where thermal fluctuations of the $\mathrm{HL}$ are evident even at zero field, $\rho_{y x}$ is always larger than $\rho_{y x}^{\text {est below } H_{c}}$ [Fig. 3(f)]. As seen from the above, the $H$ dependence of $\rho_{y x}$ changes dramatically in response to the static and dynamic character of the HL.

The connection between the magnetic state and the Hall response becomes clearer when we compare their development with both $T$ and $H$. Figure 4(a) displays the $H$ dependence of $\rho_{y x}^{\mathrm{T}}$ at various $T$. With increasing $T$, the broad negative dip structure observed below $T=20 \mathrm{~K}$ becomes confined to the low- $H$ region, and the response becomes dominated by the growing positive peak. Summarizing together the $\rho_{y x}^{\mathrm{T}}$ profiles with the SANS data 

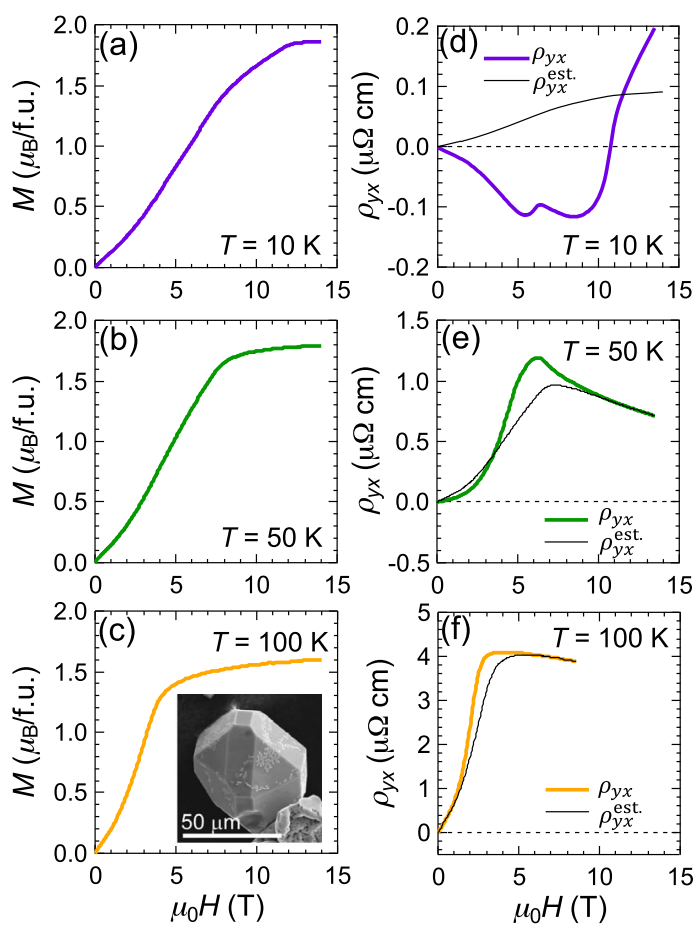

FIG. 3. (a)-(c) Magnetic-field dependence of magnetization along [111] in the thick (3 $\mu \mathrm{m})$ epitaxial (111) film at various temperatures. Inset of (c) shows scanning electron microscope (SEM) image of a MnGe single crystal. (d)-(f) Magnetic-field dependence of Hall resistivity $\rho_{y x}$ and the estimated contribution from conventional Hall response $\rho_{y x}^{\text {est }}$ in the single crystal at various temperatures.

in the phase diagram of Fig. 4(b), we find that the emergence of positive $\rho_{y x}^{T}$ correlates with the fluctuating feature of the HL. It is thus evident that the $H$ of the positive peak $\left(H_{\mathrm{p}}\right)$ correlates with the transition of the $\boldsymbol{q}$-vector alignment. Furthermore, the positive $\rho_{y x}^{\mathrm{T}}$ shows up in the $T-H$ region where diffuse scattering patterns [cyan, orange, and yellow markers in Fig. 4(b)] appear near the transition boundary of the HL transformation. In contrast, the negative $\rho_{y x}^{T}$ is confined to the low- $T$ region where the SANS pattern exhibits well-defined peaks due to the static HL [blue and red markers in Fig. 4(b)]. The above observations imply that the static and fluctuating properties of emergent monopoles are each likely origins of unusual Hall effects with opposite signs. As theoretically predicted [48] and experimentally demonstrated for a polycrystalline sample [33,35], the negative $\rho_{y x}^{\mathrm{T}}$ can be assigned to a THE induced by EMF that are present for both cubic and rhombohedral HLs [bottom panels of Fig. 4(b)]. On the other hand, the coincidence of the positive $\rho_{y x}^{\mathrm{T}}$ and the proliferation of spin-chirality fluctuations at the HL transformation can be reasonably assigned to skew scatterings of the spin-polarized conduction electrons due to the spinchirality fluctuations, as theoretically proposed in Ref. [26]. The prediction is for a THE-like response to emerge so long as there exists a correlated spin state with finite scalar spin
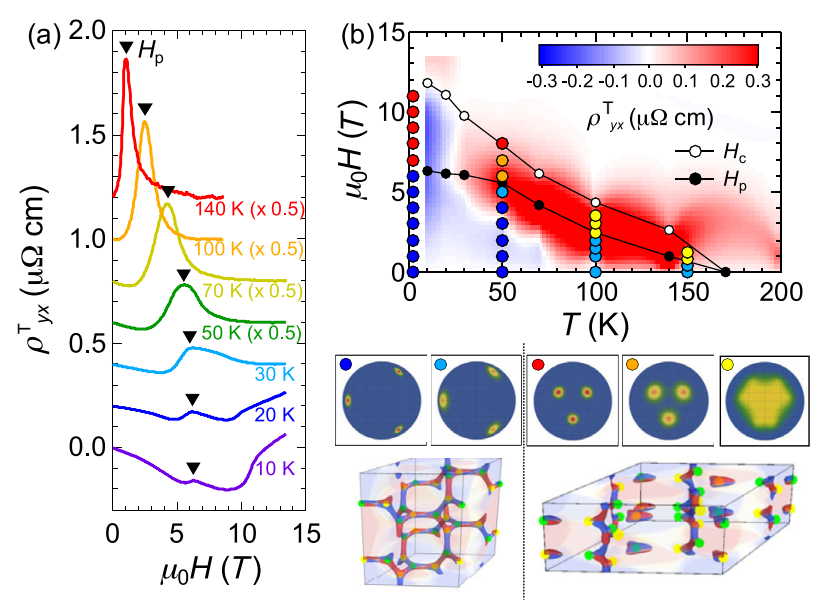

FIG. 4. (a) Magnetic-field dependence of $\rho_{y x}^{\mathrm{T}}$ in the single crystal, obtained at various temperatures. Data at adjacent temperatures are shifted vertically for clarity. Black triangles indicate the magnetic fields $H_{p}$ at the tops of positive peak structures. (b) Color map of $\rho_{y x}^{\mathrm{T}}$ and scatter plot of SANS pattern type along with critical fields $H_{p}$ and $H_{c}$. A different color marker as shown in the middle panels of (b) indicates each different category of pattern classified according to the directions and diffuseness of the scattering spots. The bottom panels are the distributions of hedgehogs (yellow dots), antihedgehogs (green dots) and emergent magnetic field (reddish and bluish regions) in the respective magnetic unit cells of the cubic (bottom left) and rhombohedral (bottom right) HL.

chirality. The present case exemplifies this situation in terms of the fluctuations of hedgehogs.

We distinguish experimentally the static and dynamic effects of emergent monopoles via their unique signatures in Hall transport. In particular, large spin-chirality fluctuations either at high $T$ or around the $H$-induced HL transformation generate a steady Hall response of opposite sign to the EMF-induced THE. The appearance of such Hall anomalies is not specific to HLs but is a general phenomenon associated with non-coplanar spin structures. It may be interesting to revisit the dynamics of topological spin singularities around the order-disorder transitions in ferromagnets [13-17]. In addition, Hall measurements on other topological spin crystals [21-23] may yield valuable information about their melting phenomena [49]. The demonstrated ability may contribute to establishing the generic properties of omnifarious topological spin textures and their excitations. The establishment of a theoretical model reproducing the complex spin texture is also left as challenges for future study.

We thank Y. Fujishiro, H. Ishizuka, Y. Motome, and S. Okumura for fruitful discussions. This work was supported by JSPS KAKENHI (Grants No. JP18K13497, No. JP20H01859, No. JP20H05155, and No. JP20H01867), JST CREST (Grant No. JPMJCR16F1 and No. JPMJCR1874), the Swiss National Science Foundation 
(SNSF) Sinergia network "NanoSkyrmionics" (Grant No. CRSII5_171003), the SNSF Projects No. 200021_ 188707 and No. 166298, and the European Research Council Project CONQUEST. We acknowledge the allocation of neutron beamtime at the Swiss Spallation Neutron Source (SINQ), Paul Scherrer Institute (PSI), Villigen, Switzerland.

*These three authors equally contributed to this work.

[1] P. A. M. Dirac, Proc. R. Soc. A 133, 60 (1931).

[2] G. Volovik, J. Phys. C 20, L83 (1987).

[3] I. Chuang, R. Durrer, N. Turok, and B. Yurke, Science 251, 1336 (1991).

[4] C. Castelnovo, R. Moessner, and S. L. Sondhi, Nature (London) 451, 42 (2008).

[5] X. L. Qi, R. Li, J. Zang, and S. C. Zhang, Science 323, 1184 (2009).

[6] P. Milde, D. Köhler, J. Seidel, L. M. Eng, A. Bauer, A. Chacon, J. Kindervater, S. Mühlbauer, C. Pfleiderer, S. Buhrandt, C. Schütte, and A. Rosch, Science 340, 1076 (2013).

[7] D. I. Khomskii, Nat. Commun. 5, 4793 (2014).

[8] M. W. Ray, E. Ruokokoski, S. Kandel, M. Möttönen, and D. S. Hall, Nature (London) 505, 657 (2014).

[9] N. D. Mermin, Rev. Mod. Phys. 51, 591 (1979).

[10] N. Nagaosa and Y. Tokura, Phys. Scr. T146, 014020 (2012).

[11] M. H. Lau and C. Dasgupta, Phys. Rev. B 39, 7212 (1989).

[12] M. Kamal and G. Murthy, Phys. Rev. Lett. 71, 1911 (1993).

[13] J. Ye, Y. B. Kim, A. J. Millis, B. I. Shraiman, P. Majumdar, and Z. Tešanović, Phys. Rev. Lett. 83, 3737 (1999).

[14] M. J. Calderón and L. Brey, Phys. Rev. B 63, 054421 (2001).

[15] Y. Lyanda-Geller, S. H. Chun, M. B. Salamon, P. M. Goldbart, P. D. Han, Y. Tomioka, A. Asamitsu, and Y. Tokura, Phys. Rev. B 63, 184426 (2001).

[16] H. Yanagihara and M. B. Salamon, Phys. Rev. Lett. 89, 187201 (2002).

[17] J. G. Checkelsky, M. Lee, E. Morosan, R. J. Cava, and N. P. Ong, Phys. Rev. B 77, 014433 (2008).

[18] V. L. Berezinskii, Sov. Phys. JETP 32, 493 (1971).

[19] J. M. Kosterlitz and D. J. Thouless, J. Phys. C 6, 1181 (1973).

[20] P. Bruno, V. K. Dugaev, and M. Taillefumier, Phys. Rev. Lett. 93, 096806 (2004).

[21] A. N. Bogdanov and D. A. Yablonskii, Sov. Phys. JETP 68, 101 (1989).

[22] S. Mühlbauer, B. Binz, F. Jonietz, C. Pfleiderer, A. Rosch, A. Neubauer, R. Georgii, and P. Böni, Science 323, 915 (2009).

[23] X. Z. Yu, Y. Onose, N. Kanazawa, J. H. Park, J. H. Han, Y. Matsui, N. Nagaosa, and Y. Tokura, Nature (London) 465, 901 (2010).

[24] C. Schütte and A. Rosch, Phys. Rev. B 90, 174432 (2014).

[25] S.-Z. Lin and A. Saxena, Phys. Rev. B 93, 060401(R) (2016).

[26] H. Ishizuka and N. Nagaosa, Sci. Adv. 4, eaap9962 (2018).

[27] T. Okubo, S. Chung, and H. Kawamura, Phys. Rev. Lett. 108, 017206 (2012).
[28] A. O. Leonov and M. Mostovoy, Nat. Commun. 6, 8275 (2015).

[29] S. Hayami, R. Ozawa, and Y. Motome, Phys. Rev. B 95, 224424 (2017).

[30] S. Okumura, S. Hayami, Y. Kato, and Y. Motome, Phys. Rev. B 101, 144416 (2020).

[31] S. Grytsiuk, J.-P. Hanke, M. Hoffmann, J. Bouaziz, O. Gomonay, G. Bihlmayer, S. Lounis, Y. Mokrousov, and S. Blügel, Nat. Commun. 11, 511 (2020).

[32] N. Kanazawa, Y. Nii, X.-X. Zhang, A. S. Mishchenko, G. De Filippis, F. Kagawa, Y. Iwasa, N. Nagaosa, and Y. Tokura, Nat. Commun. 7, 11622 (2016).

[33] N. Kanazawa, Y. Onose, T. Arima, D. Okuyama, K. Ohoyama, S. Wakimoto, K. Kakurai, S. Ishiwata, and Y. Tokura, Phys. Rev. Lett. 106, 156603 (2011).

[34] T. Tanigaki, K. Shibata, N. Kanazawa, X. Z. Yu, Y. Onose, H. S. Park, D. Shindo, and Y. Tokura, Nano Lett. 15, 5438 (2015).

[35] See the Supplemental Material at http://link.aps.org/ supplemental/10.1103/PhysRevLett.125.137202 for growth of the thick film and the single crystal of $\mathrm{MnGe}$, SANS measurement, Hall resistivity measurement, detailed temperature and magnetic field dependence of scattering intensity patterns, and comparison of transport properties between the microfabricated single crystal and the bulk polycrystal, which includes Refs. [32-34,36-46].

[36] S. Okada, T. Shishido, Y. Ishizawa, M. Ogawa, K. Kudou, T. Fukuda, and T. Lundström, J. Alloys Compd. 317-318, 315 (2001).

[37] O. L. Makarova, A. V. Tsvyashchenko, G. Andre, F. Porcher, L. N. Fomicheva, N. Rey, and I. Mirebeau, Phys. Rev. B 85, 205205 (2012).

[38] M. Deutsch, P. Bonville, A. V. Tsvyashchenko, L. N. Fomicheva, F. Porcher, F. Damay, S. Petit, and I. Mirebeau, Phys. Rev. B 90, 144401 (2014).

[39] E. Altynbaev, S.-A. Siegfried, E. Moskvin, D. Menzel, C. Dewhurst, A. Heinemann, A. Feoktystov, L. Fomicheva, A. Tsvyashchenko, and S. Grigoriev, Phys. Rev. B 94, 174403 (2016).

[40] A. Yaouanc, P. Dalmas de Réotier, A. Maisuradze, and B. Roessli, Phys. Rev. B 95, 174422 (2017).

[41] E. Altynbaev, S.-A. Siegfried, P. Strauß, D. Menzel, A. Heinemann, L. Fomicheva, A. Tsvyashchenko, and S. Grigoriev, Phys. Rev. B 97, 144411 (2018).

[42] N. Martin, I. Mirebeau, C. Franz, G. Chaboussant, L. N. Fomicheva, and A. V. Tsvyashchenko, Phys. Rev. B 99, 100402(R) (2019).

[43] Y. Fujishiro, N. Kanazawa, T. Shimojima, A. Nakamura, K. Ishizaka, T. Koretsune, R. Arita, A. Miyake, H. Mitamura, K. Akiba, M. Tokunaga, J. Shiogai, S. Kimura, S. Awaji, A. Tsukazaki, A. Kikkawa, Y. Taguchi, and Y. Tokura, Nat. Commun. 9, 408 (2018).

[44] Y. Shiomi, N. Kanazawa, K. Shibata, Y. Onose, and Y. Tokura, Phys. Rev. B 88, 064409 (2013).

[45] C. D. Dewhurst, GRASP User Manual, Technical Report No. ILL03DE01T, Institut Laue-Langevin, Grenoble, 2003, available at http://www.ill.fr/lss/grasp.

[46] N. Nagaosa, J. Sinova, S. Onoda, A. H. MacDonald, and N. P. Ong, Rev. Mod. Phys. 82, 1539 (2010). 
[47] N. Kanazawa, J. S. White, H. M. Rønnow, C. D. Dewhurst, D. Morikawa, K. Shibata, T. Arima, F. Kagawa, A. Tsukazaki, Y. Kozuka, M. Ichikawa, M. Kawasaki, and Y. Tokura, Phys. Rev. B 96, 220414(R) (2017).
[48] B. Binz and A. Vishwanath, Physica (Amsterdam) 403B, 1336 (2008).

[49] P. Huang, M. Cantoni, A. Magrez, F. Carbone, and H. M. Rønnow, Nat. Nanotechnol. 15, 761 (2020). 